\title{
Wake-up time Measurement of Wireless Sensor Node with Zero Standby-power
}

\author{
Yasunobu Takaichi, Daichi Koide, and Akira Yamawaki \\ Kyushu Institute of Technology, Kitakyushu, 804-8550 Japan \\ *Corresponding Author: yama@ecs.kyutech.ac.jp
}

\begin{abstract}
The wireless sensor network is a promising technology for environmental monitoring system, earthquake monitoring system, person tracking system, and so on. The battery replacement is one of the problems for the wireless sensor network with many battery driven sensor nodes. To tackle this problem, we have proposed and developed a power saving sensor node which does not consume any power by disconnecting the ground line by MOSFET when sleeping. However, this feature may affect waking up time of the sensor node because the proposed sensor node has to wakeup from the state without any power. In this study, the wakeup time of the proposed sensor node is measured to demonstrate our sensor node can be applied to some application of the wireless sensor network.
\end{abstract}

Keywords: wireless sensor network, standby power, battery, wake-up time

\section{Introduction}

Network technology is applied to various places and things in the world. As an example, there is the wireless sensor network, which observes the events of the outside world and processes the data observed. It is used to environmental monitoring (1), earthquake monitoring using acceleration sensor (2), and human tracking system using infrared sensor (3).

The wireless sensor network has a problem which is difficult to replace batteries on many sensor nodes.

There are the studies which use the energy harvesting without the batteries (4). However, they cannot be used at the locations without enough energy harvested.

If the battery has to be used, the sensor node used has to reduce power consumption in order to reduce the battery exchange. The normally-off on-demand system is one of the systems in order to save power (5). The sensor node cuts the power line of the analog circuits interfacing the sensors used when the sensor node is sleeping. The microcomputer on the sensor node saves electric power in its sleep mode. However, the connecting various parts can make unexpected current leakage paths increasing the standby power consumption.

To tackle this problem, we have proposed and developed a power saving sensor node which does not consume any power by disconnecting the ground line by MOSFET when sleeping (6). However, this feature may affect waking up time of the sensor node because the proposed sensor node has to wake-up from the state without any power. In this study, the wake-up time of the proposed sensor node is measured to demonstrate our sensor node can be applied to some application of the wireless sensor network.

The rest of paper is organized as follows. Section 2 briefly explains the architecture and the operation of the proposed sensor node. Section 3 explains the definition of the wake-up time of the proposed sensor node. And, Section 4 summarizes measured the wake-up time using the proposed sensor node.

\section{Proposed Sensor Node}

\subsection{Node Architecture}

Fig.1 shows the architecture of the proposed sensor node. The proposed sensor node consists of the wake-up sensor (1), a power transistor (2), a micro-controller and a wireless communication module (3). 


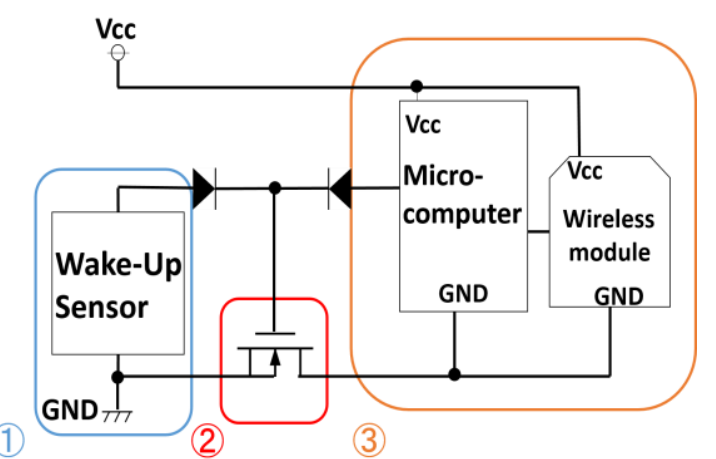

Fig. 1 The sensor node architecture

The power transistor cuts the ground line when the sensor node stays at the standby mode. Thus, the standby consumption current becomes zero.

The wake-up sensor is for energy harvesting to turn on the power transistor. When an event occurs on the wake-up sensor, the ground line is connected and the whole sensor node is energized.

The micro-controller and a wireless communication module have the role to maintain the energized and to send data.

\subsection{Node Operation}

Fig.2 shows the proposed sensor node operation.

(a)The standby state: When the sensor node is in the standby mode and no event occurs to the wake-up sensor, the power is not supplied to the proposed sensor node by MOSFET cutting the ground line. Thus, the whole of the sensor node does not consume any standby power.

(b)The wake up state: When the wake-up sensor produces voltage due to outside stimulation, the MOSFET turns on and the circuit is supplied with the electric power.

(c) Maintenance of the power supply: Only with the voltage that wake-up sensor makes, the MOSFET cannot continue to turn on. Instead, the microcomputer continues to supply voltage to the VGS of MOSFET for turning on.

(d)Data processing: The microcomputer and wireless module process the sensing data and send to master unit.

(e)The state stopping supplying voltage: After processing the data, the microcomputer stops supplying voltage to VGS right away. Thereby the circuit is not supplied voltage.

\section{Experimental Result}

\subsection{Wake-up Time}

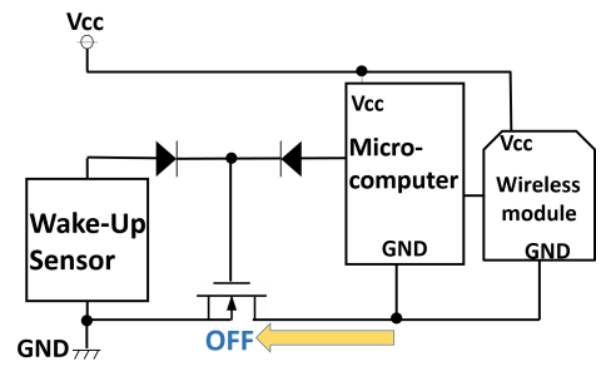

(a) The standby state

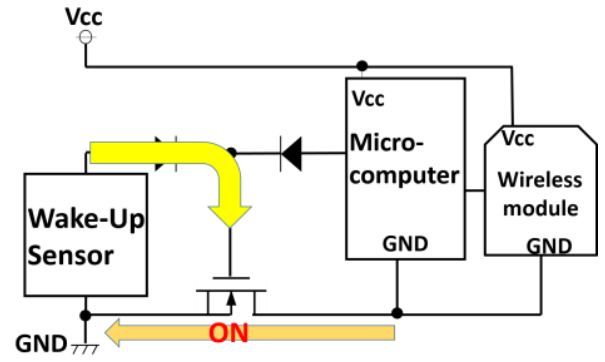

(b) The wake up state

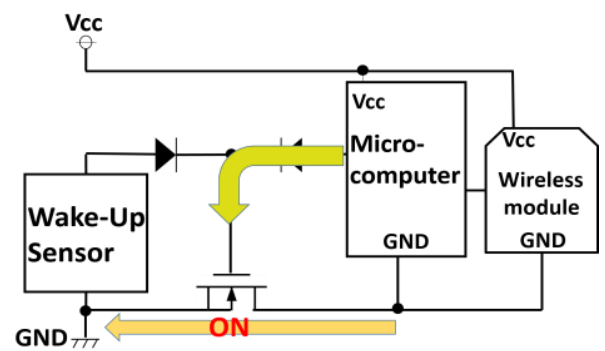

(c) Maintenance of the power supply
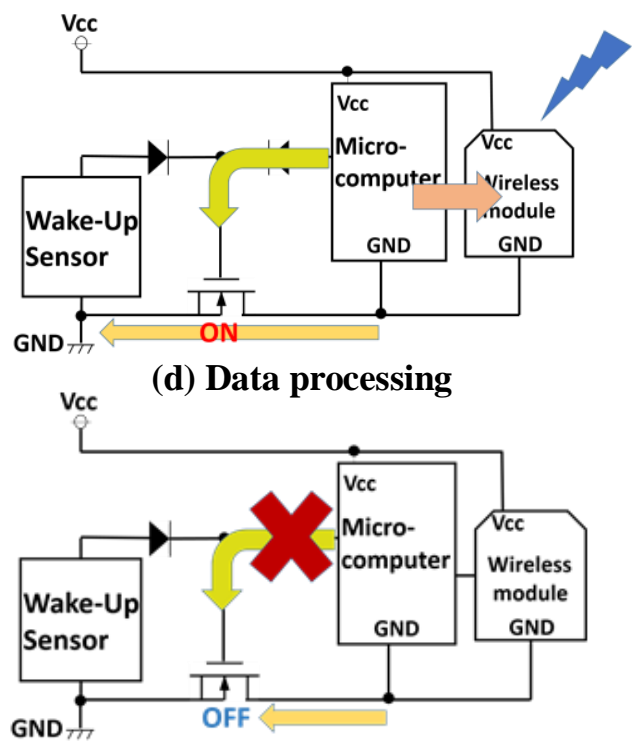

(e) The state stopping supplying voltage Fig.2.Node Operation

In this study, we define that a wake-up time is until receiving data from when electric power produced by the wake-up sensor. A breakdown of the wake-up time is shown 


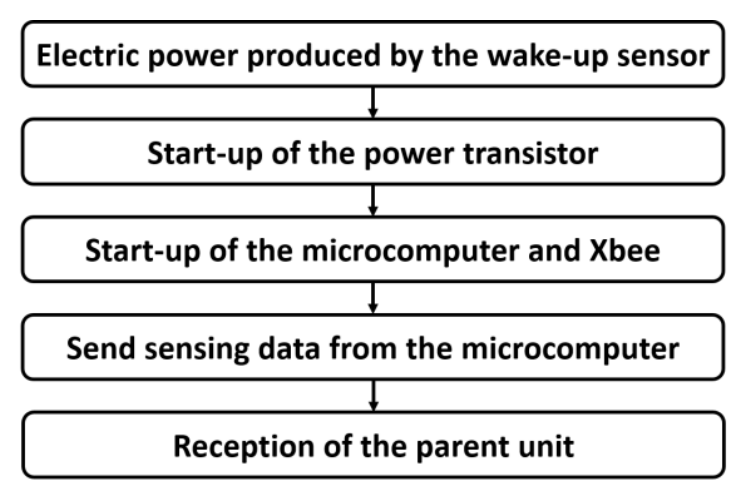

Fig.3.Definition of Wake-Up Time in Fig.3.

\subsection{Configuration of the Prototype}

Fig.4 shows the proposed sensor node configuration of prototype. We use PSoC as microcomputer, Xbee as wireless module, and piezoelectric element as wake-up sensor. We have to probe the points, $\mathrm{CH} 1$ and $\mathrm{CH} 2$, shown in Fig. 4 in order to measure the wake-up time.

\subsection{Measurement Results}

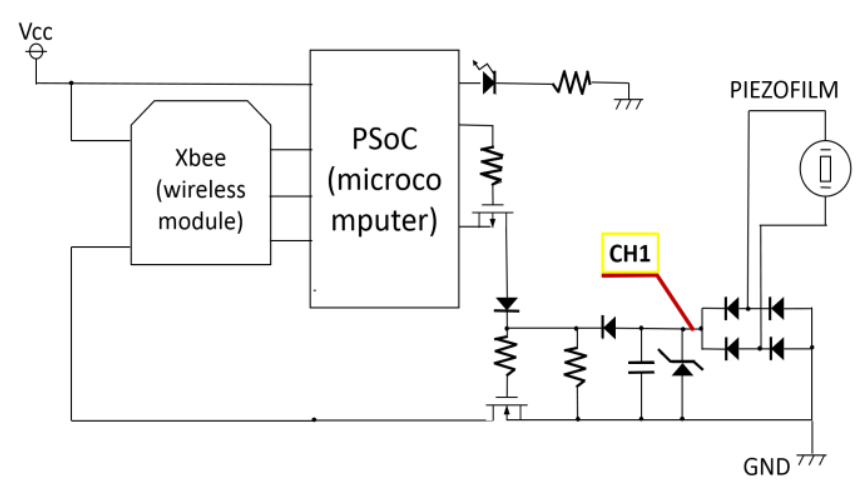

(a)Configuration of End Device

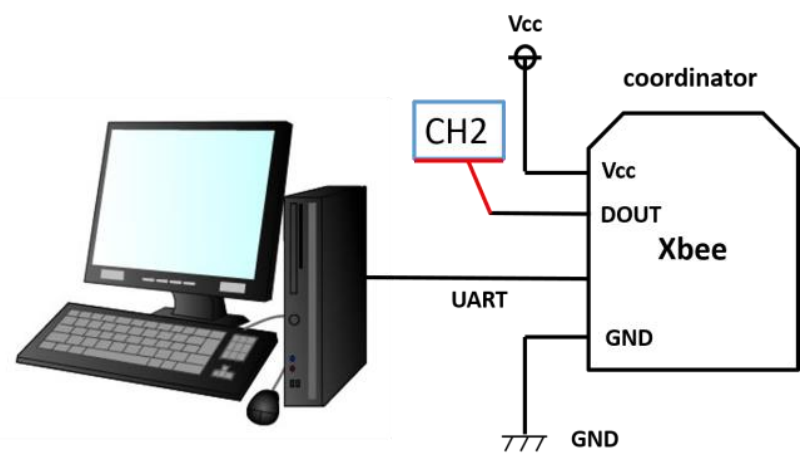

(b)Configuration of Coordinator

Fig.4. Configuration of the Prototype

Fig.5. shows the waveforms of the voltage produced by the piezoelectric element $(\mathrm{CH} 1)$ and the data signal $(\mathrm{CH} 2)$.

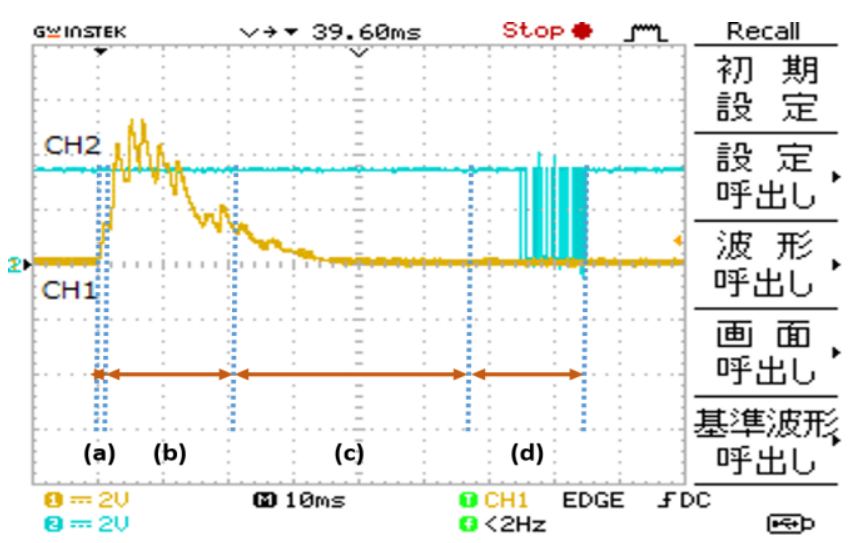

Fig.5. Waveforms the Voltage and Data signal

Table 1. Each Delay Time of the Wake-Up Time

\begin{tabular}{|c|c|}
\hline Behavior & Measurement time[ms] \\
\hline $\begin{array}{c}\text { Start-up } \\
\text { of the power transistor }\end{array}$ & (a) About 0.3 \\
\hline $\begin{array}{c}\text { Start-up } \\
\text { of the microcomputer and Xbee }\end{array}$ & (b) About 20 \\
\hline $\begin{array}{c}\text { Send sensing data } \\
\text { from the microcomputer }\end{array}$ & (c) About 36 \\
\hline $\begin{array}{c}\text { Reception } \\
\text { of the parent unit }\end{array}$ & (d) About 18 \\
\hline
\end{tabular}

Table 1 shows the measured time of each breakdown shown in Fig.3. The start-up time of the power transistor takes $0.3[\mathrm{~ms}]$. Then, the energized microcomputer and Xbee start running after 20 [ms]. The microcomputer begins to send the sensed data to the Xbee after 36 [ms]. Finally, the coordinator can get the data sent from the end device by 18 [ms]. Totally, the wake-up time defined as Fig. 3 takes about 74 [ms]. This time duration can be acceptable for many kinds of applications due to the short delay of ms order. The power-on time of the microcomputer used occupies the large part of the wake-up time as shown in Table.1.

This fact indicates that the wake-up time can be reduce more by using a microcomputer with shorter power-on time than the used one.

\section{Conclusion}

To realize a WSN with very low power consumption, we have proposed a power saving sensor node without any power when sleeping. However, the proposed sensor node worries a wake-up time because it starts from the nonenergized state. Thus, we measured a wake-up time whether our sensor node can be applied to some application of the wireless sensor network.

Experimental result shows that since wake-up time using the prototype was about 74 [ms], our sensor node can be 
applied to many kinds of applications.

As future work, the proposed sensor node is expected performance enhancement by using start-up high-speed start up microcomputer. In addition, we explore the real applications that the proposed sensor node can be employed, and we measure the power consumption on the prototype system in detail.

\section{Acknowledgement}

This work was supported in part by the JSPS KAKENHI Grant Number 26330106.

\section{References}

(1) Shin Toyota: "Environmental Monitoring System Utilizing Sensor Networks", The Institute of Electronics, Information and Communication Engineers, Vol. 9, No. 5, pp. 419-423,2006.

(2) Makoto Suzuki, Narito Kurata, Shunsuke Saruwatari, and Hiroyuki Morikawa: "Implementation and Evaluation of Earthquake Monitoring System Using Wireless Sensor Networks", The Institute of Electronics, Information and Communication Engineers, Vol. 107, No. 447, pp.65-70, 2008.

(3) Seichi Honda, Kenichi Hukui, Satoru Kurihara, and Masayuki Numao: "Multi-Person Tracking with Infrared Sensor Network", Transactions of the Japanese Society for Artificial Intelligence, Vol. 20, No. 1, pp. 102-102, 2006

(4) Kento Nitta, Ken Nagasaki, Shinzi Toda, Keizi Hirata, and Zin Matuhira: "Power consumption implementation evaluation assuming a normally-off intellectual bus stop production", The Institute of Electronics, Information and Communication Engineers, Vol. 114, No. 506, pp. 231-235, 2015

(5) Noya Ishitani, Kentarou Kobayashi, Hiraku Okada, and Masaaki Katayama, "Limitting the Sensing Frequency to Improve the Data Collection Rate in Low Power Generation for Solar-powered Wireless Sensor Networks", DPS Work Shop, Vol. 71, No. 22, pp. 1-6, 2014.

(6) Akira Yamawaki, and Seiichi Serikawa, " An Extending Method of Operable Distance for Infrared Remote Controlled Power Switch with Zero Stand-by Power", Informatics, Electronics \& Vision (ICIEV), Vol. 20, No.

3 , pp. 1-5, 2015 